\title{
Éducation au langage et valeurs du citoyen : exemples en francophonie
}

\section{Michèle Verdelhan-Bourgade}

\section{(2) OpenEdition \\ 1 Journals}

Édition électronique

URL : http://journals.openedition.org/trema/1687

DOI : 10.4000/trema.1687

ISSN : 2107-0997

Éditeur

Faculté d'Éducation de l'université de Montpellier

\section{Édition imprimée}

Date de publication : 1 octobre 1999

Pagination : 13-25

ISSN : 1167-315X

\section{Référence électronique}

Michèle Verdelhan-Bourgade, «Éducation au langage et valeurs du citoyen : exemples en

francophonie », Tréma [En ligne], 15-16 | 1999, mis en ligne le 01 octobre 1999, consulté le 20 avril 2019. URL : http://journals.openedition.org/trema/1687 ; DOI : 10.4000/trema.1687

Ce document a été généré automatiquement le 20 avril 2019

Trema 


\title{
Éducation au langage et valeurs du citoyen : exemples en francophonie
}

\author{
Michèle Verdelhan-Bourgade
}

1 Peut-on montrer, à travers des exemples d'enseignement du langage en francophonie, notamment en Afrique, de quelle manière peut se bâtir l'éducation du citoyen et quels sont les problèmes rencontrés? C'est ce que l'on va tenter de faire ici, à partir d'un corpus de manuels de français langue seconde, c'est-à-dire utilisés pour l'enseignement du français lorsque celui-ci n'est pas langue maternelle, mais pas non plus tout à fait étrangère, grâce à un usage politique, administratif ou éducatif particulier.

Pourquoi ce corpus? Certes les phénomènes apparaissent toujours mieux à une certaine distance, que celle-ci soit historique, géographique ou culturelle: c'est ainsi que de nombreuses études parmi les plus éclairantes sur les valeurs contenues dans les manuels sont le fait des historiens. La manière de traiter la distance choisie ici est un peu inhabituelle, les pays de la francophonie en langue seconde ne servant pas souvent de base à la réflexion sur l'enseignement $d u$ français langue maternelle: c'est plutôt l'inverse que l'on peut communément observer. Or dans un travail sur les rapports du savoir, du langage et de la citoyenneté, ces pays offrent un exemple très révélateur : celui de lieux dans lesquels la survie collective passe par l'éducation et l'éducation par l'enseignement du langage en français. Les conditions de vie y exigent une formation constante à un certain nombre de valeurs, le souci de construire ou de maintenir une unité nationale et une cohésion sociale rend plus sensible le rôle de la langue et de son enseignement. Remarquons d'ailleurs au passage que la question du langage dans ses rapports avec la citoyenneté resurgit à un moment où la crainte de la division sociale commence à agiter la France.

3 Pour comprendre ce que peut être une éducation citoyenne par le langage, on examinera d'abord, à partir des manuels de français langue seconde, quelles valeurs sont véhiculées par l'enseignement du langage en français. Mais l'étude des valeurs est-elle suffisante? Oui, si l'on pose, comme on le faisait au début du siècle, qu'il y a relation directe entre les valeurs présentes dans les manuels et la formation du citoyen. Non, si l'on suppose que 
les rapports entre le langage et la citoyenneté sont plus complexes, et il faut dans ce cas essayer d'en cerner les figures, pour tenter de démêler à quoi sert vraiment le langage dans la formation du citoyen.

\section{Des manuels vecteurs de « valeurs »}

4 On trouvera en fin de texte ( $c f$., Corpus des manuels utilisés) la référence complète des ouvrages utilisés, qui seront cités dans le courant de la communication sous forme abrégée.

Dans la recherche des valeurs présentes dans les manuels de langage on a été amené à repérer sommairement dans ces dernières deux grandes catégories : celles qui relèvent de la morale sociale et celles qui sont davantage liées au politique. Ce classement, loin d'être un a priori, a découlé de l'examen des ouvrages scolaires utilisés.

\subsection{Des valeurs humaines et sociales}

5 Les manuels de français langue seconde présentent fréquemment des leçons visant à la préservation de la personne physique. On trouve ainsi presque systématiquement des leçons d'hygiène : hygiène des mains, des dents, utilisation des latrines (Guide africain [...], p. 48-49), protection des aliments (eau, lait, viande) (Ibid. : Cf., ill., p. 16). Si certains aspects de l'hygiène corporelle sont véhiculés en effet par la langue maternelle ou par la religion, d'autres ne peuvent être connus des enfants que par l'intermédiaire de l'école et donc du langage en français. D'où l'insistance des manuels sur ces questions, ainsi que sur tous les sujets liés à la santé: les soins de première urgence, les vaccinations, le dispensaire. Les maladies les plus fréquentes sont expliquées, ainsi que leur prophylaxie, et l'on peut trouver des leçons sur le paludisme, le choléra et maintenant le sida!

6 D'autres leçons traitent de la préservation de l'environnement matériel. Les travaux des champs selon les saisons fournissent une réserve abondante de textes et d'illustrations propices à un travail du langage; il arrive, mais moins fréquemment, qu'on parle du jardinage et de son utilité vivrière. De même, si le danger du feu de brousse est souvent dénoncé, celui de l'abattage des arbres et des arbustes l'est rarement, malgré ses effets désastreux sur l'environnement.

7 Arrêtons-nous un instant sur ces contenus de leçons. Certains ont pour les lecteurs français un parfum d'archaïsme, nous rappelant les manuels de français du début du XX siècle, quand l'éducation linguistique et l'éducation morale et sociale ne faisaient qu'un, de manière ferme et presqu'ostentatoire. D'autres peuvent nous surprendre ou nous choquer, comme les passages sur le sida dans un livre pour le Cours moyen. Tous cependant sont liés à des urgences éducatives pour les pays concernés, que ce soit dans le domaine de l'hygiène, de la santé et de la maladie, de l'agriculture, dans des endroits encore peu industrialisés et où les épidémies peuvent se propager rapidement. Et si on parle peu de la désertification entraînée par la coupe des arbres, c'est sans doute parce que la protection de l'environnement passe après la nécessité de nourrir la population.

Cet examen pourrait nous amener à nous interroger : et nous, que faisons-nous pour nos propres urgences ? Bien sûr il n'est peut-être plus utile de marteler dans les manuels la nécessité de se laver les dents. Mais cela signifie-t-il que notre société ne connaît plus de problèmes de préservation de la personne physique et de son environnement? Ces 
problèmes, anciens ou nouveaux, ont été évacués des manuels scolaires, tournés plutôt vers le ludique, la fantaisie, l'imaginaire. Les manuels ne peuvent-ils à nouveau avoir un rôle fort de vecteur moral et social? Pourquoi faire comme si le sida était réservé à l'Afrique, la drogue aux USA, la défoliation à l'Europe de l'est et la prostitution à Manille ou à Cuba?

\subsection{Des valeurs idéologiques}

Les manuels n'en restent pas toujours à l'éducation morale et sanitaire et les valeurs transmises relèvent aussi de l'idéologie, liée tantôt à un pays, tantôt à un système politique. Il arrive fréquemment en effet que le manuel exalte la beauté d'un pays (Algérie, 4 année, p. 103), et par là la fierté d'appartenance à ce pays; parfois c'est le simple sentiment d'appartenance à une communauté qui fait l'objet de l'enseignement : c'est ainsi que Le grand Voyage de Biram [...] au Sénégal se conclut par : "Et voilà comment naquit chez le jeune Biram le sentiment d'appartenir à un grand ensemble». On note d'ailleurs dans les manuels en dix ans une évolution progressive de la présentation d'une Afrique rurale et traditionnelle à une Afrique urbaine et moderne. Là aussi il s'agit d'urgences africaines liées au développement et à ses nécessités. Mais la citoyenneté n'est-elle pas basée sur ce sentiment d'appartenance à une collectivité institutionnelle? N'est-on pas en train de regretter en France l'inexistence de ce sentiment chez toute une partie de la jeunesse scolaire, même si les circonstances politiques font évoluer l'idée de nation dans la perspective de l'Europe? Que contiennent nos manuels (hors manuels de l'éducation civique) sur ce sujet?

L'idéologie peut prendre un tour plus nettement politique et les manuels chercher à former aux lois d'un système. Un manuel angolais propose ainsi des textes faisant l'éloge du drapeau, symbole de la libération nationale, ou de l'O.D.P, une organisation d'adultes, l'Organisation de la Défense Populaire, composée de "fils du pays qui s'organisent en milices populaires pour défendre l'intégrité territoriale [et qui] luttent contre les ennemis internes et externes» (Angola; p. 175). Celui du Burkina Faso fait l'éloge des pionniers, organisation de la jeunesse, «les plus jeunes militants de la révolution» et de leur devise: "Oser lutter, savoir vaincre» (Burkina; p. 10-11). On remarquera que les pays cités sont des républiques démocratiques populaires, qui sont seuls en effet à faire une éducation politique explicite à travers l'enseignement de la langue. On la remarque d'autant plus, le décalage politique s'ajoutant au décalage géographique. Cela signifie-t-il cependant qu'elle soit superflue ailleurs?

Plus généralement, au-delà des particularismes nationaux et politiques, les manuels transmettent-ils des valeurs humaines universelles? 
OUVRAGE COLLECTIF(par un groupe d'enseignants camerounais) : Guide africain de la santé - Manuel d'hygiène - Cours préparatoire. Paris, Les Éditions Foucher - Distribution : Hatier (Paris) \& Les Éditions Africaines (Douala, Cameroun), Juillet 1988, 61 p., p. 24.

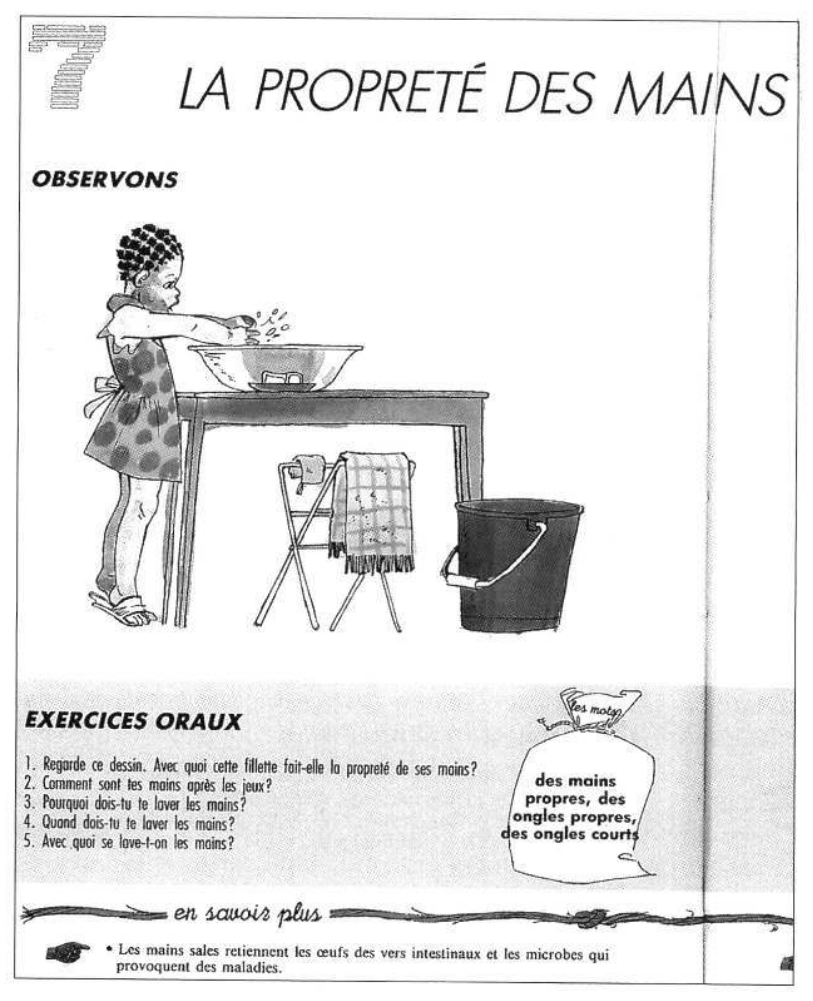

On fera en préalable deux remarques. Le financement des manuels en usage en Afrique se fait souvent par des organismes comme la Banque Mondiale, le FMI, l'UNESCO. Organismes internationaux, les payeurs définissent quelques grandes lignes qu'ils souhaitent voir diffuser dans les ouvrages. De plus, des principes généraux de politique éducative sont élaborés par des conférences internationales, comme la conférence de Jomtien, définissant la politique de l'école de base, ou la CONFEMEN. La CONFEMEN (Conférence des Ministres de l'Education Nationale) affiche un certain nombre de principes généraux et insiste notamment sur la démocratisation de l'enseignement, le partenariat, la scolarisation des filles ou la formation professionnelle et technique.

Que retrouve-t-on dans les manuels? D'une manière récurrente, l'éloge du travail, la sensibilisation aux métiers, souvent de l'artisanat ou les travaux des champs; de plus en plus souvent la scolarisation des filles, et les classes présentées dans les manuels sont mixtes. On peut noter aussi une évolution dans la présentation du rôle de la femme, du rôle traditionnel rural (le travail de la maison, celui des champs ou du jardin) à des métiers à part entière (couturière, infirmière) et même à des rôles de responsabilité (directrice d'agence de voyages, créatrice de mode, vétérinaire, in La Pirogue) : mais ces derniers exemples restent en nombre très limité.

L'éducation aux valeurs faite par les manuels de français en Afrique est donc centrée sur l'hygiène, la santé, l'école, la famille, le travail. Peut-on dire à partir de ces exemples que l'apprentissage du langage est une formation à la citoyenneté ? Ou plutôt la formation donnée par les manuels suffit-elle pour faire un citoyen et lequel? 


\section{Les rapports paradoxaux de l'apprentissage du langage et de la citoyenneté}

\subsection{Quand il ne suffit pas d'apprendre à parler français pour être citoyen}

15 L'examen des manuels fait apparaître quelques «creux » intéressants. Les exemples cidessus ont montré que la citoyenneté enseignée relevait d'une conception surtout humaine et sociale, ou civile. Or la citoyenneté c'est aussi l'exercice des droits politiques : le vote, la représentation démocratique. Les seules formes d'éducation dans ce domaine sont, comme on l'a vu, les organisations de jeunesse dans les républiques populaires. Les autres pays ne s'y intéressent-ils pas? L'âge tendre des enfants n'est-il pas un bon prétexte à reculer une formation de ce type après l'école primaire (alors que la plupart des enfants n'iront jamais dans le secondaire)?

La citoyenneté comporte également l'exercice des libertés individuelles et collectives: c'est un thème que les manuels n'abordent pas. La formation à la libre expression est rare et limitée à quelques activités dites communicatives dans les manuels les plus récents; la sécurité n'est envisagée que sous l'angle de la sécurité routière. Là encore s'agit-il de prendre en compte l'âge des enfants ou plutôt de refléter une conception de la citoyenneté dans laquelle ce genre de valeurs n'est pas fermement affirmée?

La question de l'égalité ou celle de la justice font l'objet d'un traitement plus complexe. Les manuels, surtout à travers les contes, présentent des forts et des faibles, des puissants (le roi, le lion) et des sujets, des malins et des sots. Le lièvre faible mais malin peut l'emporter sur le chameau fort mais sot. Les pauvres y sont naturellement pauvres, et les riches aussi naturellement riches : c'est un ordre des choses régulier et normal. L'égalité entre hommes et femmes n'est pas vraiment abordée, la définition des rôles respectifs restant souvent marquée par les conceptions traditionnelles.

18 Si apprendre à parler en français grâce à un manuel de langage permet à l'enfant d'être confronté à certaines valeurs, cela ne lui offre donc pas cependant la possibilité d'accéder à toute la gamme des composantes de la citoyenneté, telle que nous pouvons l'envisager. Peut-on dire que certaines valeurs ne sont pas nécessaires dans les pays considérés, ou pas urgentes? Peut-on dire qu'un manuel n'a pas de rôle à jouer dans ce domaine ? Cela aussi nous renvoie à nos propres outils d'enseignement.

\subsection{Quand il ne suffit pas d'apprendre à parler sa langue maternelle pour être citoyen}

19 Les manques constatés ne proviennent-ils pas en partie du fait que les manuels examinés sont des manuels de français langue seconde, destinés à la scolarisation en français alors que les enfants arrivent à l'école en parlant une autre langue? Le rapport entre langue maternelle et langue seconde du point de vue de la citoyenneté est un sujet délicat et complexe sur lequel on pourrait discuter longuement et dont on se contentera ici de souligner quelques aspects.

Dire que seul l'apprentissage de la langue maternelle est vecteur de citoyenneté est positif pour la fierté nationale, mais peu conforme à la réalité. Un certain nombre de 
valeurs sont transmises par l'école et l'éducation citoyenne n'est jamais laissée à la seule famille. Ne pourrait-on même parler d'éducation à une citoyenneté familiale et sociale à travers la langue maternelle et à une citoyenneté « civique » en français langue seconde ? Un apprentissage en français est porteur non seulement des valeurs nationales, mais aussi de celles véhiculées par cette langue, à travers ses usages internationaux. Reprenons la question de l'égalité des sexes : si le manuel ne vient pas contre-carrer le poids de la tradition, il n'y a aucun autre moyen pour les filles d'apprendre qu'il existe pour elles d'autres activités possibles que le travail des champs, et pour les garçons que des filles peuvent exercer ces activités. Le manuel peut véhiculer cette image plus facilement s'il est écrit en français, en raison de la distance justement que la langue seconde peut prendre avec la coutume.

Dans certains pays, les langues vernaculaires sont multiples comme les ethnies. Un exemple simple, étudié par B. Maurer $(1993)^{1}$ est donné par Djibouti, qui compte 500000 habitants et 4 langues, le français, l'arabe, le somali, l'afar. Les langues officielles sont le français et l'arabe, la scolarisation se fait en français, qui est aussi la langue de l'administration. Les enfants à l'école sont donc généralement en situation de français langue seconde, lorsque leur langue maternelle est l'arabe, l'afar ou le somali. Le manuel Le cabri et le chacal participe à l'éducation à la citoyenneté par les thèmes évoqués dans la première partie, mais aussi par la prévention de la violence ou un travail sur la presse. Dans un pays où les affrontements ethniques peuvent être violents et ils l'ont été dans un passé récent, le choix de telle ou telle langue n'est pas neutre ; le manuel en français peut alors transmettre des messages qui dans une des langues maternelles pourraient être refusés par l'autre groupe.

Un autre exemple est celui de pays à langues multiples, où se développe un apprentissage scolaire en langue nationale, par abandon du français langue seconde. Au Tchad on trouve environ 70 langues réparties en trois grands groupes, auxquelles il faut ajouter l'arabe, le peul et le français. La scolarisation se fait actuellement en français, une expérimentation est en cours pour développer un enseignement en langue nationale. Comme on ne peut scolariser dans les 70 langues parlées au Tchad, il est question de n'en choisir que 6 et on a commencé par deux ou trois, dont bien sûr celle de l'ethnie au pouvoir. La plupart de ces langues ne sont pas écrites et même si les linguistes travaillent à créer des transcriptions, elles ne donnent accès à aucune information écrite, ni livres ni journaux, à aucun contact écrit avec un groupe ou un pays voisin. Peut-on vraiment parler alors d'éducation citoyenne ? L'apprentissage scolaire du langage n'est-il pas à ce moment-là un enjeu politique bien plus que citoyen?

\subsection{Quand le langage s'oppose à la citoyenneté}

23 La Côte d'Ivoire présente le cas exemplaire de la langue "nouchi ", étudiée par Jérémie Kouadio (1990)2et Nathalie N'Guessan (1997)3. Au départ c'est une langue cryptique, liée au milieu délinquant, qui a été ensuite vulgarisée comme langue de communication des jeunes. Elle a pour base syntaxique le français, combiné avec des mots empruntés aux autres langues ivoiriennes. Ce phénomène n'est pas très éloigné dans son principe et son fonctionnement de celui repéré dans notre société sous le nom de langue des cités.

Les problèmes posés par le nouchi renvoient à une réflexion de l'ordre de la citoyenneté, comme la langue des cités. Est-ce une appropriation moderne du français par la jeunesse ivoirienne? L'apparition d'une nouvelle réalité linguistique qui permettrait de dépasser 
l'opposition entre français langue seconde et langues nationales? L'intérêt sociolinguistique du nouchi rejoindrait alors un intérêt politique.

Est-ce une langue de socialisation restreinte à un groupe donné, permettant la reconnaissance dans le groupe, la transmission des valeurs du groupe, mais ne permettant pas l'insertion dans un groupe plus large, la promotion sociale et l'activité administrative ou professionnelle? L'intérêt sociolinguistique est ici en contradiction avec les préoccupations citoyennes.

Langue défouloir ou langue ghetto? Est-ce un hasard si c'est la langue des jeunes des milieux populaires ? Le parallèle est frappant avec la langue dite des cités, et les mêmes problèmes soulevés par le nouchi se sont posés aux linguistes et aux éducateurs lors de la journée d'études «Ecole, publics, langages » en mars $1998^{4}$, consacrée pourtant à des situations bien françaises.

La préoccupation identitaire marquée par le nouchi ou la langue des cités est-elle compatible avec la formation du citoyen? Ou comment éviter que la langue par une préoccupation identitaire ne se transforme en ghetto, en langue d'exclusion?

\section{Que signifie former un citoyen par l'éducation au langage?}

\subsection{Faire prendre la parole}

Une composante de la citoyenneté est la possibilité de prendre la parole au sein d'une société. Etre citoyen c'est donc déjà développer une affirmation de soi. L'enseignement en français langue seconde, par une méthodologie proche de celle du français langue étrangère et orientée vers la communication, peut contribuer à ce développement. Ainsi, au Tchad, après quelques mois d'apprentissage du français avec un manuel basé sur une approche communicative, les enfants se montrent, de l'avis des maitres, plus éveillés, plus actifs, plus habiles verbalement. En Ethiopie, où les habitudes de communication font que l'on parle à l'enseignant les yeux baissés, le cours de français est une formation à une nouvelle norme de communication, qui suppose une autre position de soi vis-à-vis du maître.

L'importance de pouvoir dire «je» dans la parole scolaire, au lieu de s'en tenir à un commentaire des aventures d'autrui, est reconnue depuis longtemps et a conduit à toute une pédagogie de l'expression. Celle-ci a cependant ses limites. Former un citoyen n'est en effet pas synonyme de former des individualités et le rôle de l'école dans cette formation ne se résume pas à une construction du soi. La construction identitaire n'est pas du ressort exclusif de l'école, ni de l'éducation au langage. Par contre ce qui semble du ressort de l'école, c'est de permettre à l'individu d'acquérir les moyens langagiers de son insertion sociale, que son milieu d'origine, avec le langage qui y est pratiqué, ne lui aurait peut-être pas fournis. Th. Gaillat $(1998)^{5}$ cite l'exemple d'un jeune mécanicien qui reconnait lui-même qu'il ne trouve pas de travail non pas parce qu'il n'y en a pas, non pas parce qu'il manque de compétence professionnelle, mais parce qu'il parle «mal», d'une façon qui heurte les employeurs; c'est pourtant sa langue d'expression personnelle, de construction identitaire à l'intérieur de son groupe social.

30 P. Bourdieu $(1982)^{6}$ a montré depuis longtemps que prendre la parole c'est prendre le pouvoir. Beaucoup de maîtres en Afrique ou en Asie redoutent d'ailleurs le 
développement de la parole chez leurs élèves à travers des activités de communication impliquant une prise de position personnelle. On note également de multiples résistances institutionnelles à cette prise de parole : elles se manifestent dans le rappel des traditions pédagogiques, qui privilégient récitation, répétition, c'est-à-dire obéissance et soumission, dans de nombreux pays, notamment en Afrique francophone.

On peut s'insurger contre ces résistances, au nom du développement de l'individu et d'une certaine conception de la libre expression. Mais il ne faut pas oublier qu'un apprentissage du langage se fait dans un certain contexte institutionnel, politique et social qu'il faut prendre en considération. La prise de parole n'a pas forcément de valeur en soi, mais seulement dans une visée d'insertion sociale.

\subsection{Former au sens}

32 Former au langage, au-delà de la prise de parole, c'est former au sens, et cette formation est capitale pour l'intégration sociale et la pratique citoyenne. Elle permet d'éviter que le langage tourne à vide, comme c'est la pratique courante en CB (Citizen Band) ou maintenant sur les «chats » dont le désert langagier est souvent navrant, réduit à une fonction phatique élémentaire. Savoir dire du sens c'est aussi ne pas faire la confusion entre langage ornemental et langage référentiel, entre les discours ronflants et attractifs mais creux et ceux qui sont porteurs d'informations ou de propositions; c'est une situation courante en politique comme en pédagogie. C'est aussi éviter les confusions conceptuelles, repérer les facilités de langage, les glissements d'un mot à l'autre qui recouvrent des glissements de la pensée: du savoir aux savoirs, de la langue à la communication, de l'égalité à la parité, de la liberté à la facilité de choix... Les exemples abondent de l'exercice citoyen d'une vigilance sémantique.

\subsection{Forger des structures mentales}

33 Les structures mentales se forment par les expériences du sujet, et l'expérience langagière est une expérience fondamentale. On sait bien par exemple que chaque langue comporte une conception spécifique du temps. Mais tout langage s'inscrit dans le temps, dans une linéarité que la langue exploite à sa façon. Or l'observation du langage des cités, ou de celui des adolescents, montre que c'est un langage haché, déstructuré, procédant par flashes successifs, bousculé comme par un refus de la linéarité, des étapes syntaxiques du langage courant. Ce n'est pas une question de correction ou d'incorrection syntaxique; il s'agit plutôt d'une démarche mentale liée à un mode langagier.

Les apprentissages scolaires du langage influencent les modes de pensée, notamment à travers la compréhension et la réalisation des consignes. Une consigne fréquente, par exemple, est celle de "classer». Qu'est-ce que classer? Ce peut être distribuer par catégories («classe les noms d'animaux et de plantes»), ranger par ordre de grandeur ( « classe du plus petit au plus grand»), répartir dans l'espace sous forme de colonnes, de tableaux. Cela induit des activités mentales spécifiques, une certaine représentation de l'espace, un certain type d'ordre à apporter dans un ensemble d'éléments. Or certaines langues n'apprennent pas à classer. En ce sens on peut dire qu'apprendre à parler dans une langue donne une certaine structuration mentale qui n'existe pas forcément dans une autre. Ce qui donne une autre dimension à la question de l'apprentissage scolaire en langue maternelle ou en français langue seconde. 


\subsection{Donner accès au savoir}

Autant les savoirs sont multiples et variés, présents dans toutes les situations de vie (on parle d'ailleurs de savoir être, de savoir faire, et plus du tout de savoir vivre, ce qui est légèrement paradoxal), autant le savoir est global, bien que modulable: une personne peut être de peu de savoir, ou de grand savoir. Il y a des degrés dans le savoir, il y a de la diversité dans les savoirs.

Les savoirs peuvent-ils se construire sans le langage ? Cela resterait à étudier mais c'est peu probable; encore que le rôle du langage dans l'apprentissage de la planche à voile soit assez limité ! Le savoir, lui, ne passe-t-il pas nécessairement par le langage?

Langage et éducation a l'environnement.

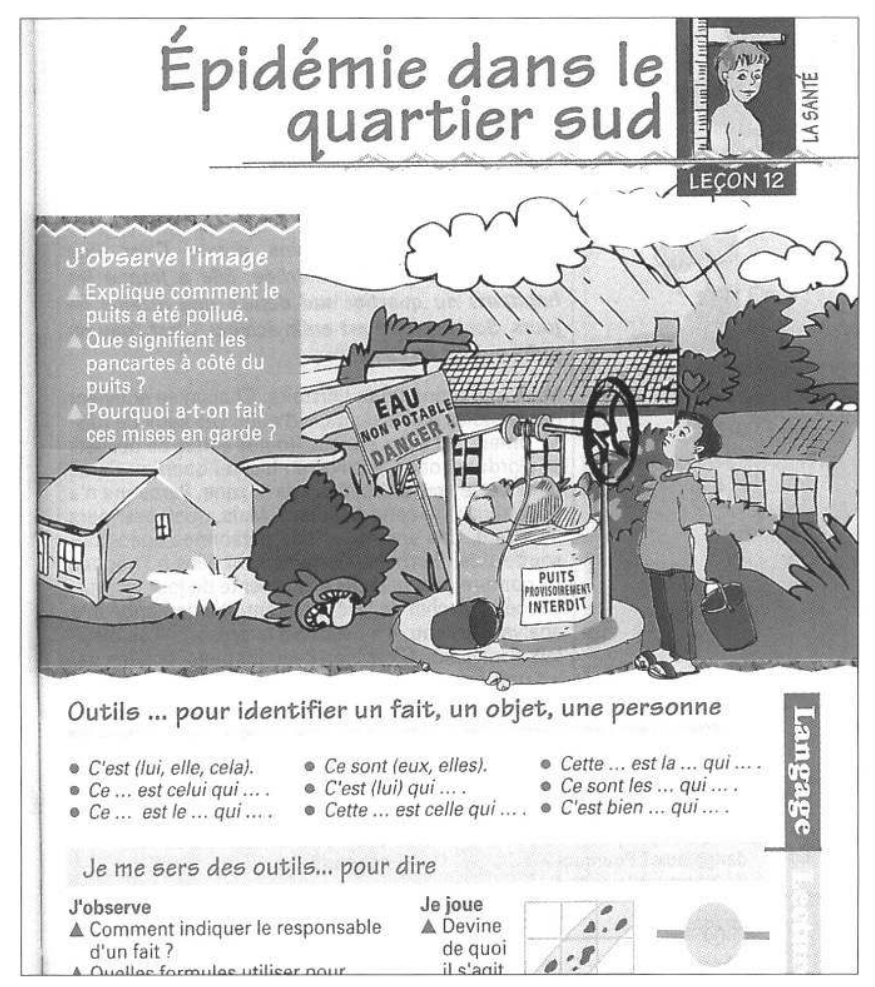

Manuel d'apprentissage du français en classe bilingue, AUPELF-UREF.

IREFA : Ici et ailleurs, Méthode de Français Universel - 4e année. Paris, Hachette - Edicef, 1997, 143 p., p. 69.

La francophonie l'a bien compris qui, de Haïti au Vietnam, donne à ses élites, par le biais des classes bilingues, l'accès au savoir universel. L'écrit, sous forme papier ou électronique, contient la bibliothèque du monde et si dans certains pays on bloque l'accès des chercheurs au courrier électronique ou à l'Internet, c'est bien parce qu'accéder à cette bibliothèque c'est accéder au pouvoir né de la connaissance.

$\mathrm{Au}$ bout de ce parcours rapide en francophonie, que peut-on dessiner comme perspectives à une éducation citoyenne du langage ? Former un citoyen par le langage se décline en plusieurs strates qu'on a essayé d'identifier au moins sommairement. On en conclura que la réhabilitation de l'éducation au langage ne doit pas se faire seulement dans la recherche de la construction identitaire ou le développement personnel de 
l'expression, mais dans une visée d'insertion sociale et d'acquisition du savoir. On laissera enfin la parole à un manuel de français ${ }^{7}$, et à un poète d'expression française, qui sait dire en quoi le langage est porteur de citoyenneté.

\section{Alphabétisation}

A quoi servent mes poèmes

Si ma mère ne sait me lire?

Ma mère a vingt ans

Elle ne veut plus souffrir

Ce soir elle viendra Epeler mes lettres

Et demain elle saura

Ecrire

Emancipation

A quoi servent mes poèmes

Si mon père ne sait me lire?

Mon père $a$ cent ans

Il n'a pas vu la mer

Ce soir il viendra

Epeler mes lettres

Et demain il saura

Lire

Dignité

A quoi servent mes poèmes

Si mon copain ne sait me lire?

Mon copain n'a pas d'âge

Il a vécu dans les prisons

Ce soir il viendra

Epeler mes lettres

Et demain il saura

Crier

Liberté

Rachid BOUDJEDRA. « Pour ne plus rêver », Alger, SNED.

\section{BIBLIOGRAPHIE}

\section{Corpus des manuels utilisés}

I.R.E.F.A. : La Pirogue - CE1, CE2, CM2. Paris, EDICEF, 1994 à 1996.

Premier livre de français. $4^{\mathrm{e}}$ année fondamentale. Alger, Institut pédagogique national, 1988.

Guide africain de la santé - CP. Paris, Hatier, 1988.

Le beau voyage de Biram à travers le Sénégal. Abidjan, NEA, 1975. 
Le cabri et le chacal - CI, CP. Djibouti, Éd. : CRIPEN, EDICEF \& Ministère de l'Education Nationale. 1993-1994.

Livre de lecture, $3^{\mathrm{e}}$ année. Éd. : Institut Pédagogique du Burkina \& Ministère de l'enseignement de base et de l'alphabétisation de masse. IPB-Vuibert, 1989.

Mon premier livre de français. Enseignement de base, $7^{\mathrm{e}}$ classe. Luanda (Angola), Ministère de l'Education, 1980.

EKOTTO EBOLO A. : Mon livre unique de français. Langage-lecture. Cameroun, CEPER-EDICEF, 1988. Sidi et Rama - CI et CP. Dakar (Sénégal), Éd. : INEADE \& Ministère de l'Education Nationale. 1991.

\section{NOTES}

1. MAURER B. : Le français en République de Djibouti. Aspects linguistiques et sociolinguistiques Thèse. Montpellier, Université Montpellier III, 1993.

2. KOUADIO N'GUESSAN J. : « Le nouchi abidjanais, naissance d'un argot ou mode passagère ? ", in Des langues et des villes. Collection Langue et développement,

3. N'GUESSAN N. : Le parler des jeunes en Côte d'Ivoire: le nouchi - Mémoire de maîtrise (sous la dir. de B. Maurer). Montpellier, Université Montpellier III, 199

4. Coord. : MAURER B. : «Actes du colloque : Ecole, publics, langages», in Discours Didactiques, №3. Montpellier, DIDAXIS-DIPRALANG - Université Montpellier III, 1999

5. GAILLAT T. : Scolarisation des enfants de migrants arabophones. Préalables à une didactique intégré Thèse. Montpellier, Université Montpellier III, 19

6. BOURDIEU P. : Ce que parler veut dire. Paris, Fayard

7. La Pirogue - CM2. Paris, EDICEF, 1996, p

\section{RÉSUMÉS}

A travers l'étude de quelques outils d'enseignement du langage en francophonie, notamment en Afrique, on s'interroge sur la manière dont peut se bâtir l'éducation du citoyen et quels obstacles elle rencontre.

Through the study of a few language teaching tools in French speaking countries worldwide, specifically in Africa, the author addresses the issue of the means by which the education of the citizen may be built and of the obstacles it runs up against.

\section{INDEX}

Mots-clés : français, francophonie, langue seconde, manuel scolaire

Keywords : French, french speaking countries worldwide, school textbook, second language 
AUTEUR

MICHĖLE VERDELHAN-BOURGADE

IUFM de Montpellier et Université de Montpellier II, équipe Didaxis 